\title{
Do Cochrane reviews provide useful information to guide policy and practice? The experience of the Cochrane Drugs and Alcohol Group
}

\author{
M. Davoli* and L. Amato \\ Department of Epidemiology, Regional Health Authority Lazio, Cochrane Drugs and Alcohol Group, Via S. Costanza 53, 00198 Rome, Italy
}

\begin{abstract}
This contribution reviewed the experience of the Cochrane Drugs and Alcohol Group (CDAG) in terms of production of evidence to guide policy and practice. By December 2010, the group had published 55 reviews, with 299 authors involved and 744 primary studies included out of 2114 studies considered for inclusion. $90 \%$ of the studies included were randomized controlled trials (RCTs). Considering the 'Implication for practice' section of each review, 31\% interventions were classified as to do, $11 \%$ as do not do it, $52 \%$ to do only in research and for $6 \%$ a final judgment was impossible because the reviews included no studies or only one study. These proportions varied according to the type of substance of abuse studied; interventions judged as to do were $42 \%$ for alcohol, $32 \%$ for opioids, $12 \%$ for psychostimulants, $33 \%$ for poly drugs, and for prevention. The reviews produced by the CDAG provide evidence on effectiveness of several interventions, and identify areas of uncertainty, where more primary research is needed.
\end{abstract}

Key words: Alcohol, drug abuse, randomized controlled trial, systematic reviews.

\section{Background}

The relevance of the drug and alcohol problem in terms of frequency and health and social impact is quite pronounced. Substance use disorders are associated with a wide range of serious health, social and economic complications. The life expectancy of alcohol and drug users is often significantly lower than that of the general population (Wahren et al. 1997; Price et al. 2001; Sørensen et al. 2005), with a great impact on the mortality of young adults (Bargagli et al. 2006). Different interventions are offered for the prevention and treatment of substance use and dependence. The choice is often guided by common sense, intuition, experience, beliefs or ideology and not always by evidence. Clinicians and policy makers need accessible, up-to-date, objective evidence regarding the effectiveness of different interventions.

The Cochrane approach puts much emphasis on the rigor of method, independence and transparency, and historically relied on reviewers' interest in specific topics and individual commitment. As soon as the potential use of Cochrane reviews was recognized, particularly in UK, Canada and Australia, substantial amount of investment has been made in trying to

* Address for correspondence: Dr. Marina Davoli, Director of Department of Epidemiology, Regional Health Authority Lazio and co-ordinating editor of the Cochrane Drugs and Alcohol Group, Via S. Costanza 53, 00198 Rome, Italy.

(Email: davoli@asplazio.it) produce reviews that were not only of good quality but were also relevant for different stakeholders (clinicians, consumers, policy makers, etc.). While there is a wide range of literature showing that the quality of Cochrane systematic reviews is consistently better that non-Cochrane reviews (Jadad et al. 2000; Olsen et al. 2001; Moja et al. 2005; Delaney et al. 2007; Moher et al. 2007; Jørgensen et al. 2008; Tricco et al. 2009), there is less evidence about the relevance and ability of Cochrane reviews to meet the needs of the different stakeholders. Actually some papers have been recently published raising issues of potential limits of Cochrane reviews (Mandel et al. 2006; Lang et al. 2007; Pagliaro et al. 2010).

The aim of this paper is to review the experience of the Cochrane Drugs and Alcohol Group (CDAG) in terms of production of evidence to guide policy and practice. For this purpose, we analyzed the contribution of the systematic reviews published by the CDAG as of 31 December 2010 in terms of: scope of the group, coverage of topics, body of primary researched reviewed and eventually results of reviews in terms of sufficient evidence to inform practice and research.

\section{What is in the Cochrane Library}

The CDAG, as part of the Cochrane Collaboration, aims to produce, update and disseminate systematic 
reviews of trials on the prevention, treatment and rehabilitation of problematic drug and alcohol use. CDAG was founded in 1998 and has an editorial base in Rome (Davoli \& Ferri, 2000; further information is available at http://www.cdag.cochrane.org).

Cochrane reviews are produced internationally, a total of 299 authors have published with the CDAG: 185 from the European Union, 29 from Australia, 33 from Asia, 29 from North America, 10 from South America, 8 from South Africa and 5 from the Middle East. The systematic reviews published by CDAG are based primarily on randomized controlled trials (RCTs) and controlled clinical trials that describe an active intervention (including prevention, treatment and rehabilitation) aimed at reducing the potential for harm or the actual harm directly related to the use of different dependence-producing substances; the inclusion of other study designs, however, is considered in limited circumstances (Amato et al. 2011).

By December 2010, the CDAG published 55 reviews covering pharmacological and psychosocial treatments of opioid (21 reviews), alcohol (12 reviews), cocaine and other psychostimulant (10 reviews), poly drug (4 reviews) and cannabis, benzodiazepine, inhalants and metaqualone (1 review each) abuse or dependence. The effectiveness of preventive interventions across different substances was considered in four reviews (see Table 1).

One of the main features of the Cochrane Systematic Reviews is the comprehensiveness of the search, which in fact entails an explicit transparent search strategy to find both published and unpublished trials (Higgins \& Green, 2008); for this purpose, CDAG created and maintains a specialized register of trials on the evaluation of treatment effectiveness. When the group was created, in 1998, there was no previous knowledge of the body of primary research available to conduct systematic reviews. Actually, the body of primary research on the effectiveness of interventions for alcohol and drug addiction proved to be quite substantial. As of January 2011, the register contained 7150 references to the studies.

The number of trials considered for inclusion in the 55 published reviews represent about one-third of the overall number of potentially available trials. Moreover, out of a total of 2114 trials considered for inclusion, only 744 studies were included (35\%), with a total of 241802 participants (see Table 1). Although the inclusion criteria can vary between reviews and be attributed, in part, to the sensitivity of the search strategy, the proportion of studies that satisfy the criteria is low overall.

Despite the considerable number of trials carried out on the treatment of addiction, our findings seem to

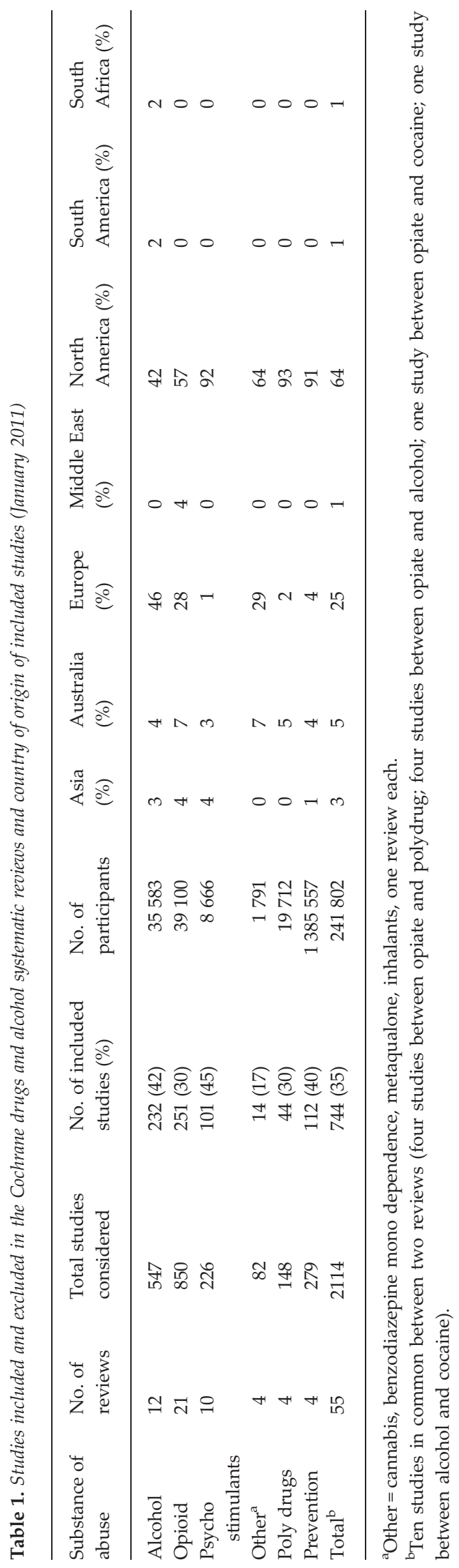


confirm that only a few of them contribute to the cumulative knowledge on the effectiveness of interventions (Chalmers, 1998) (see Table 1).

In the reviews published by the group, only $10 \%$ were not RCTs and were included in 3 out of the 55 reviews. Two of these reviews considered maintenance treatments for opioid dependence, and the reasons for inclusion were that they considered long-term outcomes, such as mortality, difficult to be properly analyzed in RCTs due to power limitations. The other non-RCT studies were included in one review on preventive interventions in which non-RCTs often represent the only available source of evidence.

\section{Challenges for the Cochrane reviews}

The extent to which a Cochrane review can draw conclusions about the effects of an intervention depends on whether the data and results from the included studies are valid. Quality of evidence is part of the judgment provided by Cochrane reviews: for each study included in a Cochrane review, quality is judged in relation to risk of bias for sequence generation, allocation concealment and blinding; eventually, when results of a Cochrane review come from studies with high risks of bias, the quality of the produced evidence will be described as low.

However, systematic reviews should evaluate and take into account not only the internal validity (i.e., the extent to which systematic errors or bias are avoided) of each trial included but also the applicability and generalizability or external validity (i.e., whether the results of a trial can be reasonably applied to a definable group of patients in a particular setting in routine practice) (Dekkers et al. 2009).

The main threat to external validity comes from the clinical setting, and the social and cultural context in which the studies were conducted, and this is particularly true in the field of addiction, where these contexts can actively affect the overall treatment outcome.

Primary studies included in these reviews, as shown in Table 1, were conducted in North America (64\%), Europe (25\%), Australia/New Zealand (5\%), Asia (3\%), the Middle East (1\%), South America (1\%) and South Africa (1\%). The distribution, however, was heterogeneous across substances of abuse; for example, studies conducted in North America varied, respectively, from $93 \%$ and $92 \%$ for polydrug abuse and psychostimulants, respectively, to $42 \%$ and $57 \%$ for alcohol and opioid dependence, respectively.

Finally, systematic reviews could be improved in the future by improving the applicability of the results in clinical practice. A recent article (Ahmad et al. 2010) assessed the methods and reporting of information on the applicability of trial results in systematic reviews and found that the applicability is poorly reported or taken into account. In order to ameliorate the applicability of results, in the future, authors must identify which applicability items are important (according to the type of treatment evaluated) and should be collected and reported.

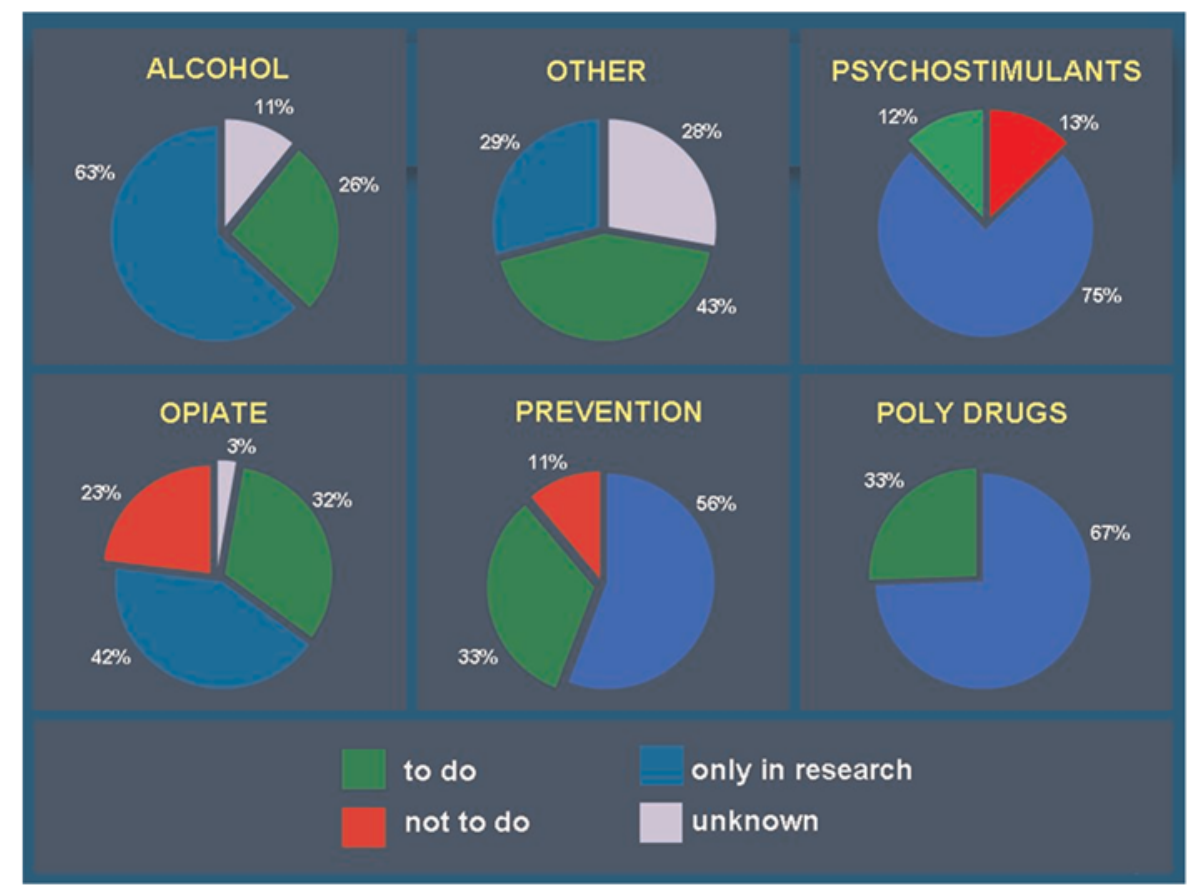

Fig. 1. Evidence of effectiveness of interventions considered in the CDAG reviews. Cochrane Library 2.2011. 


\section{Are results of Cochrane reviews useful to inform clinical practice?}

In order to measure the extent to which the published Cochrane reviews in the field of drug and alcohol do provide evidence for each intervention evaluated in the systematic reviews, we considered the 'Implication for practice' section of the Cochrane review and, following the classification suggested by NHS and UK Cochrane Centre (http:/www.library.nhs.uk/qipp/), we classified the interventions as sufficient evidence to: implement the intervention into clinical practice (Do it), to avoid the intervention into clinical practice (Do not do it) and insufficient evidence (Only for research).

Based on these criteria, for the interventions considered in the published reviews, 25 (31\%) were classified as to do, $9(11 \%)$ as do not do it, $25(52 \%)$ to do only in research and for $5(6 \%)$ the judgment was impossible because the reviews included no studies or only one study.

These proportions varied according to the type of substance of abuse studied; for example, the interventions that were judged as to do were $26 \%$ for alcohol, $32 \%$ for opioids, $12 \%$ for psychostimulants, $33 \%$ for polydrugs and for prevention (see Fig. 1). These results should be considered cautiously, remembering that they referred only to interventions, comparisons and outcomes considered in the studies included in the reviews, and we are aware that the evidence presented is not thorough and definitive. Furthermore, the assessment of the methodological quality of included studies showed relevant weaknesses in the information available to judge their quality, which is not considered in this summary of available evidence.

\section{Conclusions}

Systematic reviews published by the CDAG cover a wide range of substances and interventions, and provide evidence on effectiveness of several interventions.

Providing evidence of effectiveness, however, is only the first step to reduce the gap between practice and policy. Even though Cochrane reviews in the field of addiction were already used to inform clinical guidelines (Prodigy, 2005; Australian National Drug Strategy, 2006; NICE, 2006), it is only after the recent development of the GRADE method (Guyatt et al. 2008) that global guidelines based on Cochrane reviews have been published (WHO, 2009). The main features of the GRADE method that facilitated the development of global guidelines for the treatment of addiction is the explicit identification of critical outcomes and the separation between judgment on quality of evidence and strength of recommendation. A method that provides a transparent and pragmatic way of combining evidence to value judgment and preference to formulate clinical recommendations is of particular use in the field of drug addiction where several cultural and social constraints are involved, and production of evidence-based guidelines have always been problematic.

However, if Cochrane reviews are developed 'to provide the type of information that is needed by physicians to make clinical decisions' there is still some progress to be made at least in the way they are presented. Most reviews are too lengthy containing many sections, most of which describing the adherence of the review to the standardized methodology of the Collaboration, which can make their consultation timeconsuming with respect to the clinical content of interest for clinicians; a more user-friendly format of Cochrane reviews should be probably developed in the near future to overcome this limitation.

The other side of the coin that should be considered is the potential role of Cochrane reviews to inform the research agenda; there are no empirical data describing the extent to which this occurs, at least in the field of addiction. Cochrane reviews, in fact, do also underline areas of uncertainty, and eventually the high proportion of trials excluded in the reviews highlight a major issue of relevance of primary research in this field. Eventually, Cochrane systematic reviews should also be considered as a tool to inform the research agenda in terms of setting priorities, identifying areas of uncertainty and promoting multicenter high-quality studies addressing questions that contribute to progressing knowledge on the effectiveness of treatment and, consequently, meet the needs of patients, their care givers and policy makers.

Eventually, there are several questions to which our reviews are not providing answers; this may frustrate the confidence of physicians in the usefulness of Cochrane reviews to answer relevant clinical questions, in these cases, when evidence from randomized trials is lacking or insufficient, the opportunity of evaluating the evidence coming from non-randomized studies should be further explored.

\section{Role of Funding Sources}

Funding for this study was provided by the Department of Epidemiology, Regional Health Authority Lazio.

\section{Contributors}

Marina Davoli and Laura Amato wrote the manuscript.

\section{Conflict of Interest}

Both authors declare that they have no conflict of interest pertaining to the manuscript. 


\section{References}

Ahmad N, Boutron I, Dechartres A, Durieux P, Ravaud P (2010). Applicability and generalisability of the results of systematic reviews to public health practice and policy: a systematic review. Trials 26, 11-20.

Amato L, Davoli M, Vecchi S, Ali R, Farrell M, Faggiano F, Foxcroft D, Ling W, Minozzi S, Chengzheng Z (2011). Cochrane systematic reviews in the field of addiction: What's there and what should be. Drug and Alcohol Dependence 113, 96-103.

Australian National Drug Strategy (2006). National clinical guidelines and procedures for the use of buprenorphine in the treatment of opioid dependence. Available at http:// www.health.gov.au

Bargagli AM, Hickman M, Davoli M, Perucci CA, Schifano $\mathrm{P}$, Buster M, Brugal T, Vicente J, for the COSMO European Group (2006). Drug related mortality and its impact on adult mortality in eight European countries. European Journal of Public Health 16, 198-202.

Chalmers I (1998). Systematic reviews: insufficient evidence on which to base medicine. British Journal of Obstetrics and Gynecology 105, 1235-1236.

Davoli M, Ferri M (2000). The cochrane review group on drugs and alcohol. Addiction 95, 1473-1474.

Dekkers OM, von Elm E, Algra A, Romijn JA, Vandenbroucke JP (2009). How to assess the external validity of therapeutic trials: a conceptual approach. International Journal of Epidemiology 39, 89-94.

Delaney A, Bagshaw SM, Ferland A, Laupland K, Manns B, Doig C (2007). The quality of reports of critical care meta-analyses in the Cochrane Database of Systematic Reviews: An independent appraisal. Critical Care Medicine 35, 589-594.

Guyatt GH, Oxman AD, Kunz R, Vist GE, Falck-Ytter Y, Schünemann HJ (2008). What is 'quality of evidence' and why is it important to clinicians? British Medical Journal 336, 995-998.

Higgins JPT, Green S (2008). Cochrane Handbook for Systematic Reviews of Interventions. Version 5.0.2 [updated September 2009]. The Cochrane Collaboration. Available at http://www.cochrane-handbook.org

Jadad AR, Moher M, Browman GP, Booker L, Sigouin C, Fuentes M, Stevens R (2000). Systematic reviews and meta-analyses on treatment of asthma: critical evaluation. British Medical Journal 320, 537-540.

Jørgensen AW, Maric KL, Tendal B, Faurschou A, Gøtzsche PC (2008). Industry-supported meta-analyses compared with meta-analyses with non-profit or no support: differences in methodological quality and conclusions
BMC. Medical Research Methodology 8, 60. doi:10.1186/ 1471-2288-8-60.

Lang A, Edwards N, Fleiszer A (2007). Empty systematic reviews: hidden perils and lessons learned. Journal of Clinical Epidemiology 60, 595-597.

Mandel D, Littner Y, Mimouni FB, Lubetzky R (2006). Conclusiveness of the Cochrane Neonatal Reviews: a systematic analysis. Acta Paediatrica 95, 1209-1212.

Moher D, Tetzlaff J, Tricco AC, Sampson M, Altman DG (2007). Epidemiology and reporting characteristics of systematic reviews. Public Library of Science Medicine 4, e78. doi:10.1371/journal.pmed.0040078.

Moja LP, Telaro E, D'Amico R, Moschetti I, Coe L, Liberati A (2005) Assessment of methodological quality of primary studies by systematic reviews: results of the metaquality cross sectional study. British Medical Journal 330, 1053.

NICE (2006). National Institute of Clinical Excellence Technology Appraisal Guidance 114. Methadone and buprenorphine for the management of opioid dependence. Available at http://www.nice.org.uk

Olsen O, Middleton P, Ezzo J, Gøtzsche PC, Hadhazy V, Herxheimer A, Kleijnen J, McIntosh H (2001). Quality of Cochrane reviews: assessment of sample from 1998. British Medical Journal 323, 829-832.

Pagliaro L, Bruzzi P, Bobbio M (2010). Why are Cochrane hepato-biliary reviews undervalued by physicians as an aid for clinical decision-making? Digestive and Liver Disease 42, 1-5.

Price RK, Risk NK, Murray KS, Virgo KS, Spitznagel EL (2001). Twenty-five year mortality of US servicemen deployed in Vietnam: predictive utility of early drug use. Drug and Alcohol Dependence 64, 309-318.

Prodigy (2005). Guidance for the use of methadone for the treatment of opioid dependence in primary care. In: National Health Service Clinical Knowledge's Summaries. Prodigy. Available at http://cks.library.nhs.uk

Sørensen HJ, Jepsen PW, Haastrup S, Juel K (2005). Drug-use pattern, comorbid psychosis and mortality in people with a history of opioid addiction. Acta Psychiatrica Scandinavica 11, 244-249.

Tricco AC, Pham B, Brehaut J, Tetroe J, Cappelli M, Hopewell S, Lavis JN, Berlin JA, Moher D (2009). An international survey indicated that unpublished systematic reviews exist. Journal of Clinical Epidemiology 62, 617-623.

Wahren CA, Brandt L, Allebeck P (1997). Has mortality in drug addicts increased? A comparison of two hospitalized cohorts in Stockholm. International Journal of Epidemiology 27, 1219-1226.

WHO (2009). Guidelines for the psychosocially assisted pharmacological treatment of opioid dependence. Available at http://www.who.int 